\title{
Efficacy of the combined use of bevacizumab and irinotecan as a postoperative adjuvant chemotherapy in colon carcinoma
}

\author{
TOMOAKI MIZOBE, YUTAKA OGATA, HIDETSUGU MURAKAMI, YOSHITO AKAGI, \\ NOBUYA ISHIBASHI, SHINJIROU MORI, TERUO SASATOMI and KAZUO SHIROUZU \\ Department of Surgery, Kurume University School of Medicine, Japan
}

Received April 7, 2008; Accepted May 22, 2008

DOI: 10.3892/or_00000036

\begin{abstract}
We evaluated the efficacy of anti-human VEGF antibody (bevacizumab) with or without irinotecan (CPT-11) against lung metastases in which neovascularization was already induced, as a postoperative adjuvant therapy using orthotopically implanted colon cancer in rat. The high VEGF productive KM12SM human colon cancer cells were injected into the cecal wall. At 5 weeks after the injection, the cecum was removed including the tumor. Then, $5 \mathrm{mg} / \mathrm{kg}$ of bevacizumab and $40 \mathrm{mg} / \mathrm{kg}$ of CPT-11 were administered, alone or in combination, intravenously once a week for 3 weeks, from day 15 after the cecal removal. The results show that the incidences of macroscopic and/or microscopic lung metastases in the bevacizumab-alone group (B) and in the combination group $(C)$ were significantly lower $(B, p=0.001$ and $C, p=0.037$ ) than that in the control group at day 35 after the cecal removal. The number of lung metastases in $\mathrm{B}$ was $0.8 \pm 0.8(\mathrm{p}=0.024)$ and in $\mathrm{C} 2.4 \pm 1.8(\mathrm{p}=0.060)$, each value lower than the $12.4 \pm 4.2$ of the control group. The growth of a subcutaneously implanted tumor was significantly inhibited in the combination group compared to either the CPT-alone $(p=0.003)$ or the bevacizumab-alone groups $(p=0.027)$. Apoptosis was significantly $(\mathrm{p}<0.001)$ induced in the combination group. In conclusion, a beneficial effect of bevacizumab against postoperative lung metastases may be expected even after the establishment of neovascularization in metastatic foci in nude rat. The results from the present subcutaneously implanted tumor model suggested that a higher efficacy may be expected when bevacizumab is combined with the cytotoxic agent CPT-11, compared to bevacizumab alone, against tumors with a variety of VEGF production levels in clinical situations.
\end{abstract}

Correspondence to: Dr Yutaka Ogata, Department of Surgery, Kurume University School of Medicine, 67 Asahi-machi, Kurume City, Fukuoka 830-0011, Japan

E-mail: yogata@med.kurume-u.ac.jp

Key words: colon cancer, bevacizumab, irinotecan, angiogenesis, postoperative adjuvant therapy, lung metastasis, orthotopic xenograft

\section{Introduction}

Colorectal cancer remains one of the leading causes of cancerrelated deaths in the world. The mainstay treatment for colorectal cancer with curative intent is surgical resection. In node-positive or stage III patients, surgery alone offers curability to $\sim 50 \%$ of patients treated (1). Thus, addressing the high risk of recurrence necessarily involves the use of adjuvant chemotherapy, immunotherapy or molecular targeting therapy after surgical removal of the primary lesion. Worldwide, the infusion of 5-fluorouracil plus leucovorin (5-FU/LV) combination chemotherapy has been considered to be standard for stage III colon cancer for the past decade (2). Recently, FOLFOX which has oxaliplatin added to a bolus plus infusion 5-FU/LV was reported to be more effective than 5-FU/LV in stage III colon cancer (3).

Angiogenesis plays a pivotal role in tumorigenesis and metastases (4). Tumor angiogenesis is a complex process based on the concept that a tumor requires a vascular blood supply to grow beyond 1 or $2 \mathrm{~mm}$ (5). Tumors that do not establish a neovascular supply may remain dormant for a long time. The transition of a tumor from the 'avascular' or 'prevascular' phase to the 'vascular' phase accompanied by an increase in growth and a metastatic potential is termed the 'angiogenic switch' $(6,7)$. The switch is believed to be stimulated by an increase in the expression of proangiogenic factors and by a decrease in antiangiogenic factors. It is also believed to be a key event for the establishment of postoperative recurrence. Vascular endothelial growth factor (VEGF) is a key molecule in the angiogenic process, and has a role in endothelial cell migration, proliferation, vascular permeability and endothelial cell apoptosis $(8,9)$. Several studies have reported a correlation between an increased VEGF expression and a greater risk of recurrence in cancer patients $(10,11)$. Therefore, given its role in the growth and development of metastasis and its relatively low expression levels in healthy adult tissues, VEGF is considered to be an attractive target for anticancer therapy, even in an adjuvant setting.

The VEGF humanized monoclonal antibody bevacizumab and/or its murine equivalent A4.6.1 has demonstrated an antitumor effect from its administration as a single agent or in combination with cytotoxic agents in preclinical models $(12,13)$. Preclinical studies have suggested that combining bevacizumab with chemotherapy and radiotherapy may result in synergistic antitumor activity (12). These findings 
are being validated in a clinical setting. Notably, the efficacy of bevacizumab combined with chemotherapy, using irinotecan (CPT-11) and 5-FU/LV, was recently determined in a phase III trial of patients with advanced colorectal cancer (13). However, its efficacy as adjuvant therapy in colorectal cancer is still being investigated.

We previously reported on the efficacy of MMI270 (matrix metalloproteinase inhibitors) on postoperative lung metastases via antiangiogenesis using orthotopically implanted colon cancer. However, the antitumor effects of MMI270 were minimal when neovascularization had already become induced in the metastases after removal of the colon cancer (14). Thus, we have postulated that cytotoxic anticancer drugs should be combined with antiangiogenic agents in such a clinical adjuvant setting. The present study was designed to evaluate the antitumor effects of anti-VEGF antibody bevacizumab combined with CPT-11 against postoperative lung metastases in orthotopically implanted colon cancer in nude rat. In particular, we identified a synergistic effect of bevacizumab and CPT-11 after the lung metastases developed neovascularization. We also investigated the antitumor effects and mechanisms of bevacizumab combined with CPT-11 using xenograft in the subcutis.

\section{Materials and methods}

Drugs. Bevacizumab was a kind gift from Genentech (South San Francisco, CA, USA). CPT-11 was a gift from Yakult Honsha (Tokyo, Japan). CPT-11 solution was freshly prepared in $0.9 \%$ saline at a concentration of $1 \mathrm{mg} / \mathrm{ml}$.

Cell culture. The human colon carcinoma cell line KM12SM, which produces a high level of VEGF in monolayer culture (supernatant: $2822 \mathrm{pg} / \mathrm{ml} / 10^{6} / 48 \mathrm{~h}$, unpublished data), was kindly provided by Dr M. Nakajima (Johnson \& Johnson K.K., Tokyo, Japan). The tumor cells were harvested from subconfluent cultures by 5-min treatment with trypsin-EDTA (Invitrogen, Tokyo, Japan). The dislodged cells were first washed in RPMI-1640 (Invitrogen) supplemented with 10\% fetal bovine serum, and re-suspended in phosphate buffer saline (PBS) for injection. Only single cells in suspension with $>90 \%$ viability were used for the injections.

Animals. Male F344nJCL/rnu/rnu rats, aged 4 weeks, were purchased from CLEA Japan (Tokyo, Japan). The rats were maintained in a laminar-flow cabinet under specific pathogenfree conditions and were used for experiments when they were 5 weeks old. The rats were maintained in facilities according to the regulations and standards of the Kurume University School of Medicine.

\section{Tumor xenografts and assessment for antitumor effects}

Tumor growth inhibition on implanted colon cancer in the subcutis. A total of $1 \times 10^{7} \mathrm{KM} 12 \mathrm{SM}$ cells/PBS was transplanted into the subcutis of the dorsal skin in nude rat. The maximum tumor diameter was set at 5-10 mm, then CPT-11, bevacizumab or their combination was administered intravenously at a dosage of 10,20 and $40 \mathrm{mg} / \mathrm{kg}$ in CPT-11 (up to half the dosage of the $\mathrm{LD}_{50}$ of $83.6 \mathrm{mg} / \mathrm{kg}$ ), and $5 \mathrm{mg} / \mathrm{kg}$ in bevacizumab once a week for 3 weeks. We calculated the body weight of the rat each week, and these data were used as an indicator of side effects. The tumor size was measured each week using calipers, and the tumor volume was calculated by the formula [(maximum tumor diameter $)^{2} \mathrm{x}$ minimum tumor diameter/2]. We then resected the tumor at 5 weeks after beginning the drug administration, and the tumor was fixed by $10 \%$ formalin for histological and biological examination.

Inhibitory activity against postoperative lung metastases. The rat was anesthetized with ether, and the abdomen prepared for sterile surgery. A small incision $(1.5 \mathrm{~cm})$ was made, and the cecum was exteriorized. A total of $2 \times 10^{6}$ cells $/ 0.02 \mathrm{ml}$ PBS of viable KM12SM cells was injected into the cecal wall from the serosal side using a 30-gauge needle. The cecum was returned to the abdominal cavity, and the wound was closed with 3-0 vicryl surgical sutures (Ethicon Inc., Somerville, NJ, USA). At 5 weeks after the injection, the rat was anesthetized and operated on as described above, and the cecum with a growing tumor was exteriorized. After confirming that the implanted tumor had grown $>5 \mathrm{~mm}$ in size, the cecum, including the lymph nodes around the meso-appendix, was carefully removed using an Endopath Endocutter (Ethicon Endo-surgery Inc., Cincinnati, OH, USA). After removal of the cecum, the wound was closed with 3-0 vicryl surgical sutures.

Based on the serial changes in the morphology of lung metastases determined in our previous study (14), we administered $40 \mathrm{mg} / \mathrm{kg}$ of CPT-11 alone, $5 \mathrm{mg} / \mathrm{kg}$ of bevacizumab alone or their combination intravenously at days 15, 22 and 29 after tumor removal. We assumed that day 15 corresponded to the time whereby the neovascularization had already become induced in the metastatic foci. The rats were sacrificed and autopsied at day 35 after the removal of the cecum. The lung was processed to count the tumor nodules on its surface and for a histological examination using hematoxylin and eosin staining (H\&E). The lung metastases were classified into three degrees: two-plus (++) for metastases recognized macroscopically on the surface of the lung, one-plus (+) for metastases detected only microscopically and minus (-) for no metastasis (Fig. 1) (14). Moreover, we calculated the body weight of the rat each week, and used this calculation as an indicator for any adverse side-effects.

Immunohistochemistry for PCNA labeling index and microvessel density. The dorsal subcutaneous tumor was fixed by formalin and embedded into paraffin. Serial $3 \mu \mathrm{m}$ sections were cut from each block. One section was stained by $\mathrm{H} \& \mathrm{E}$, a second was immunostained for CD31 and a third was immunostained for PCNA. Immunoreactivities were determined using the avidin-biotin peroxidase complex method (Vector Laboratories, CA, USA) using anti-rat CD31 (Chemicon, USA) and anti-human PCNA (Santa Cruz Biotechnology, USA) at 1:200 dilution as the primary antibody. Hematoxylin was used as the counterstain. The negative controls used all reagents except for the primary antibody. Positive staining of the small tubular formation for CD31 was defined as a macrovessel and the microvessel density (MVD) was assessed as the average number of vessels per $1 \mathrm{~mm}$ square, over three areas, at x200 magnification. The PCNA labeling index (PI) was assessed as the average percentage of positively stained cells among 500-1000 tumor cells, over five random areas, at x200 magnification. 
a)

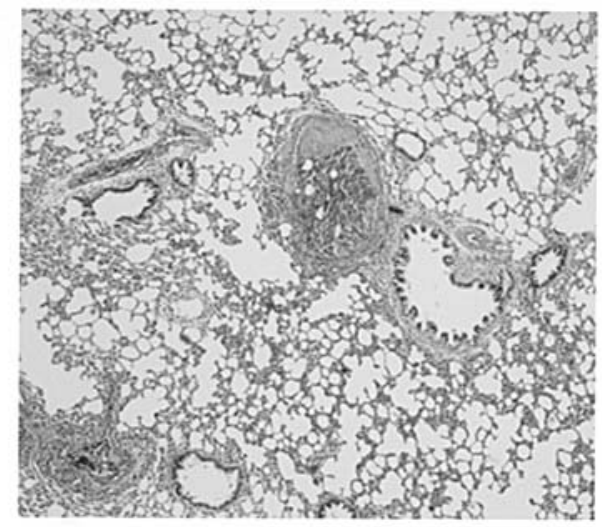

c)

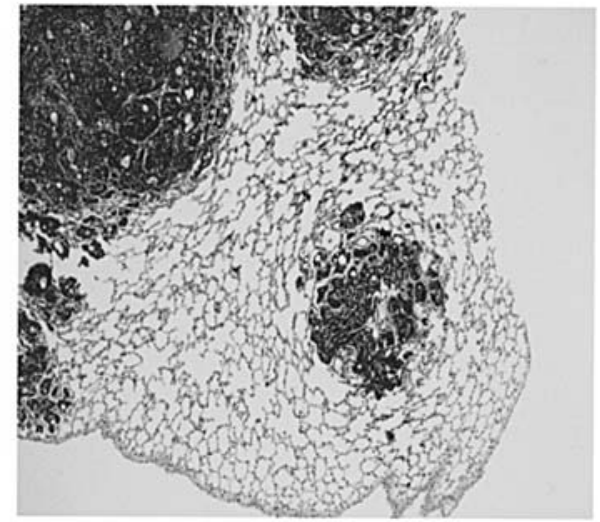

b)

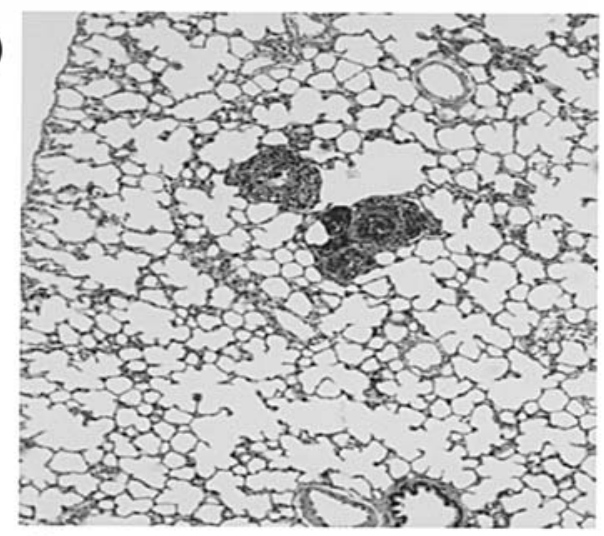

d)

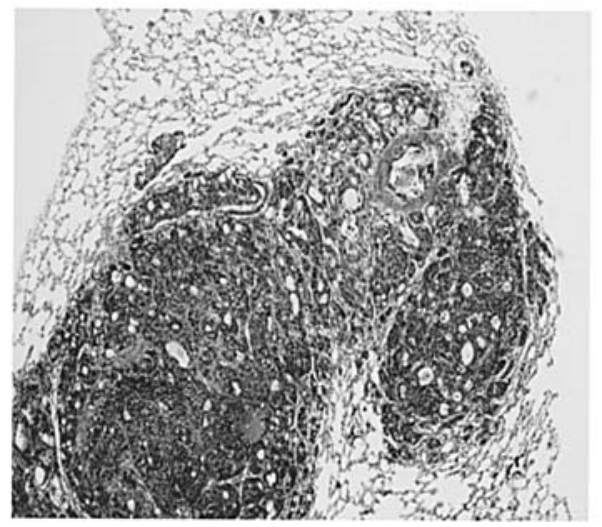

Figure 1. Degree of lung metastasis. Lung metastasis was defined by macroscopic and histological examinations. The lung metastases were classified into three degrees: two-plus (++) for metastases recognized macroscopically on the surface of the lung (c and d), one-plus (+) for metastases detected only microscopically (a and b) and as minus (-) for no metastasis.

Terminal-deoxynucleotidyl-transferase-mediated dUTPbiotin nick end-labeling (TUNEL) assay. TUNEL staining allows for the in situ detection of apoptotic cells. After deparaffinization and rehydration, the lung tumor section was incubated in $20 \mu \mathrm{g} / \mathrm{ml}$ of proteinase $\mathrm{K}$ for $15 \mathrm{~min}$ at room temperature. After rinsing in PBS for $5 \mathrm{~min}$, endogenous peroxidase activity was blocked by incubating the section in $2 \% \mathrm{H}_{2} \mathrm{O}_{2}$ for $5 \mathrm{~min}$ at room temperature. Following rinsing in PBS, each slide was covered with an equilibration buffer (in situ apoptosis detection kit, Takara Bio Inc., Otsu, Japan), and incubated for $15 \mathrm{~min}$ at room temperature. Apoptotic cells were then identified using immunoperoxidase detection system. The apoptosis index (AI) of a rat was scored as the mean number of the apoptotic tumor cells per field at 200fold magnification under light microscopy, over 3 random fields of the lung metastases.

Statistical analysis. The data were analyzed using the $\chi^{2}$ test, Fisher's test and Student's t-test. The tumor volume was analyzed using two-way repeated ANOVA. $\mathrm{P}<0.05$ was considered statistically significant. Analyses were computed using the Stat View v. 5.0 software (SAS institute Inc, USA).

\section{Results}

Growth inhibition of the implanted tumor in the subcutis. CPT-11 inhibited the growth of the implanted tumor by KM12SM cells in nude rat in a dosage-dependent manner (Fig. 2a). In particular, the intravenous $40 \mathrm{mg} / \mathrm{kg} /$ week of
CPT-11 significantly $(\mathrm{p}=0.036)$ inhibited tumor growth compared with the control. Thus, $40 \mathrm{mg} / \mathrm{kg} /$ week was found to be the recommended dosage of CPT-11 to be used for further studies. The intravenous administration of bevacizumab $(5 \mathrm{mg} / \mathrm{kg} /$ week $)$ significantly $(\mathrm{p}=0.011)$ inhibited tumor growth compared with the control (Fig. 2b). Moreover, the tumor growth in the combination group treated with $40 \mathrm{mg}$ / $\mathrm{kg}$ of CPT-11 and $5 \mathrm{mg} / \mathrm{kg}$ of bevacizumab was significantly inhibited compared with the control $(\mathrm{p}=0.002)$ (Fig. 2c), the CPT-alone group $(\mathrm{p}=0.003)$ or the bevacizumab-alone group $(\mathrm{p}=0.027)$ (Fig. 2d). No treatment related weight loss, bleeding or wound-healing problems were found (data not shown).

Microvessel density (MVD), PCNA labeling index (PI) and apoptosis index (AI). To investigate the antitumor mechanism of the combination therapy of bevacizumab and CPT-11, we evaluated the MVD, PI and AI in the implanted colon cancer in the subcutis on day 35 after the beginning of drug administration (Figs. 3 and 4). The MVD was significantly lower in the combination $(\mathrm{p}<0.001)$ and bevacizumab-alone group $(\mathrm{p}<0.001)$, compared with the control group. The PI was significantly lower in the combination group than in the control group $(\mathrm{p}<0.001)$, whereas no difference in PI was seen between the bevacizumab-alone or CPT-alone groups, and the control group. The AI in the combination group was significantly $(\mathrm{p}<0.001)$ higher than that in the control group, although no difference was found between the bevacizumabalone or the CPT-alone groups, and the control group. 
a

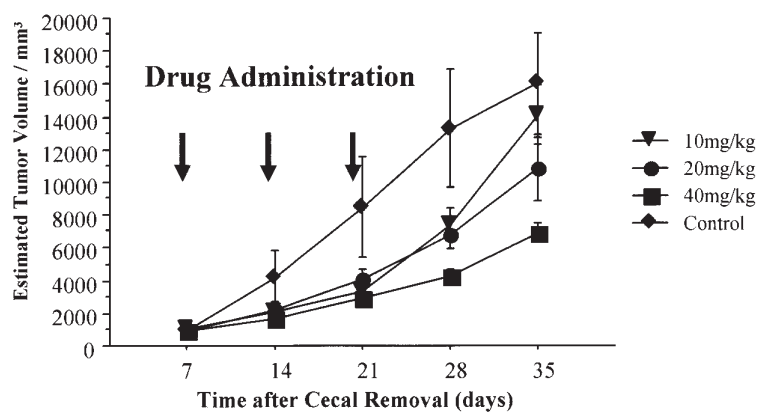

CPT-11 alone

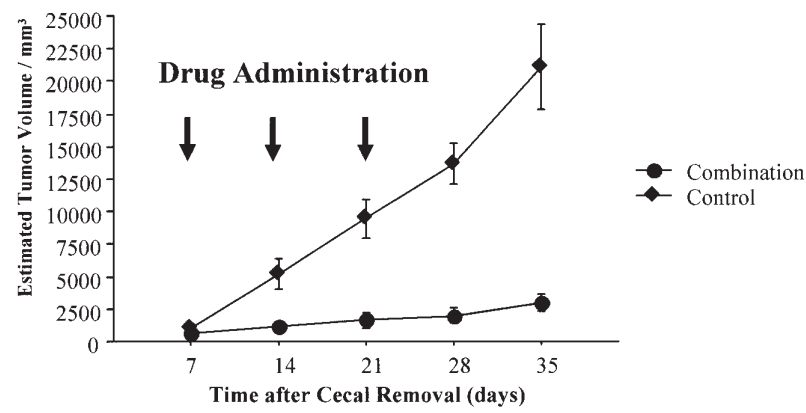

Combination of CPT-11 and Bevacizumab b

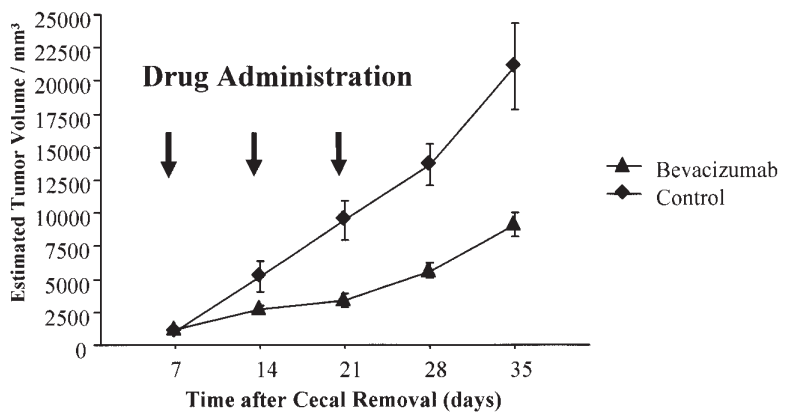

d

Bevacizumab alone

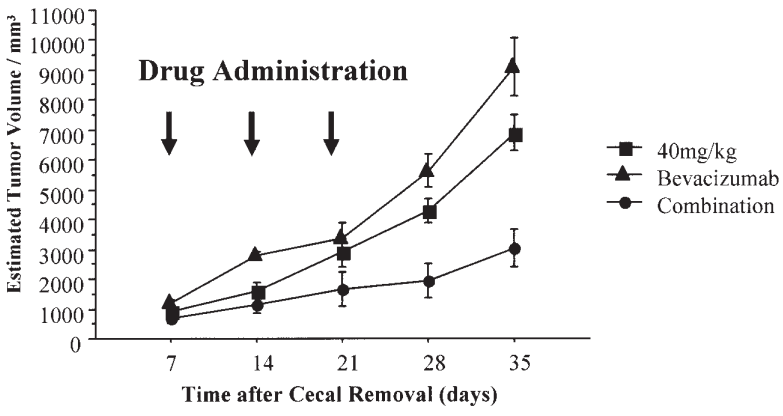

Combination Therapy and Mono-therapy

Figure 2. Growth curves of the subcutaneous tumor implanted by KM12SM cells in nude rat. The maximum tumor diameter was set at 5-10 mm, then CPT-11, bevacizumab or their combination was administered intravenously once a week for 3 weeks.

\section{Control}

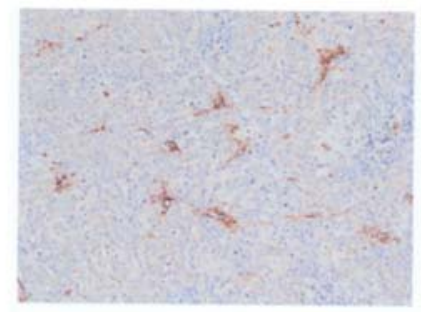

PCNA

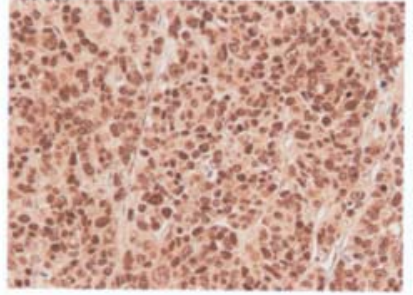

CD31 (1)

TUNEL

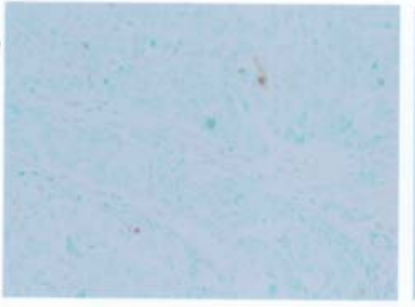

CPT-11

(40 mg/kg)
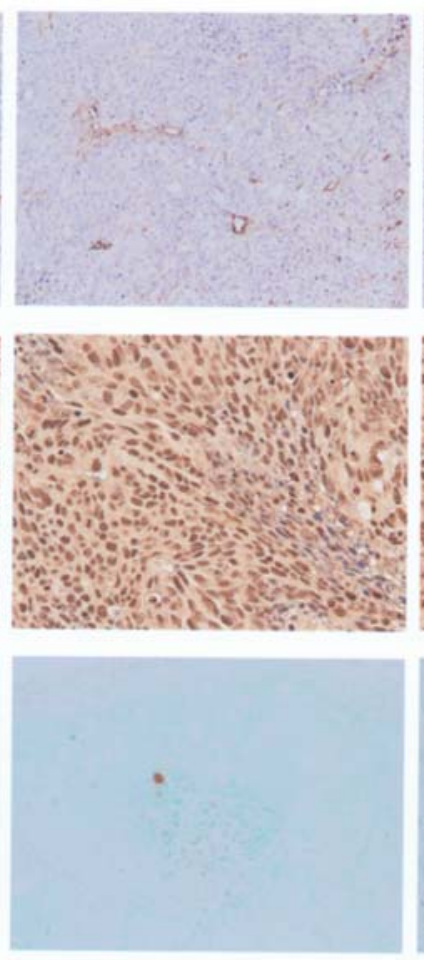

Bevacizumab
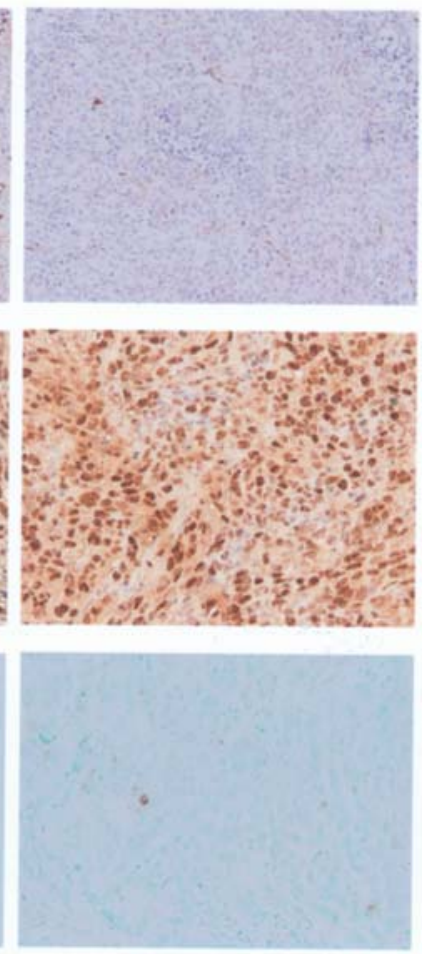

Combination
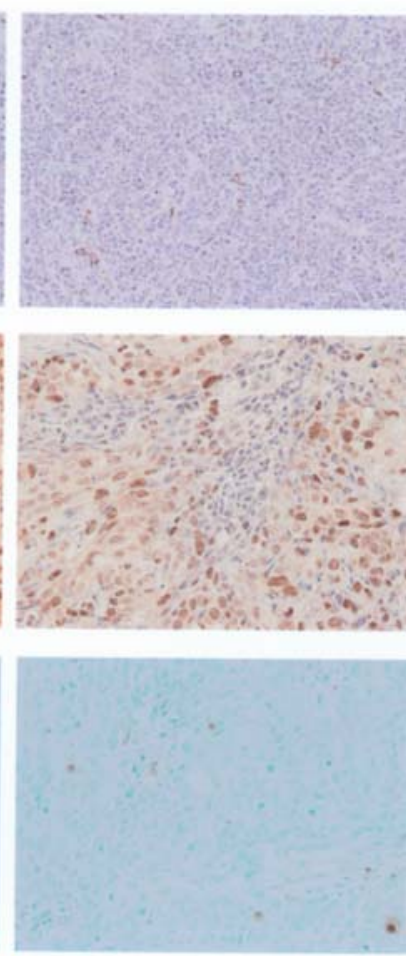

Figure 3. TUNEL staining and immunohistochemical staining for CD31 and PCNA using a subcutaneously implanted tumor. 


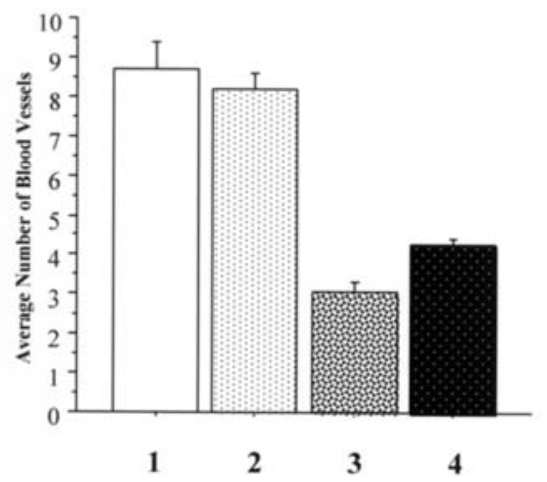

A) Microvessel Density (MVD)

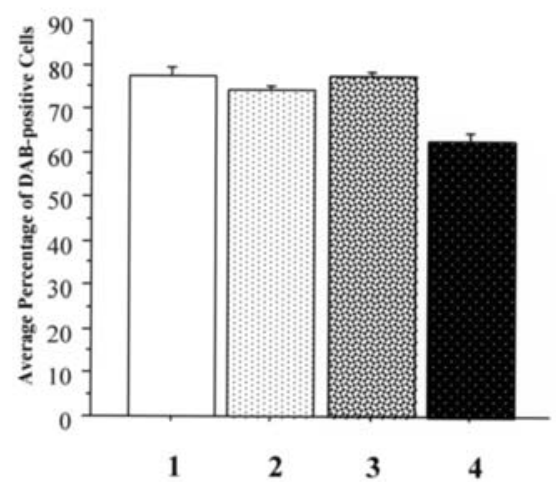

B) Proliferation Index

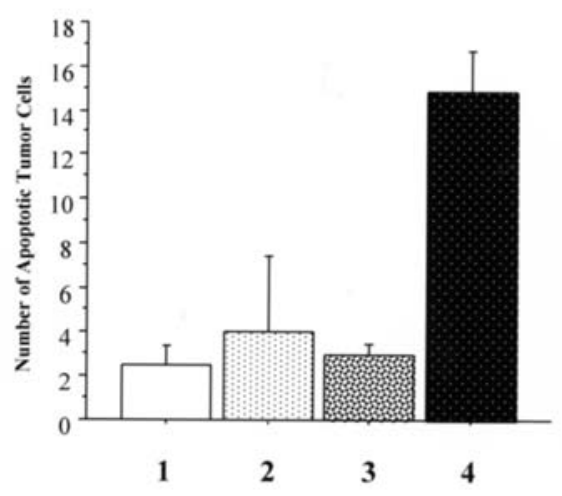

C) Apoptosis Index

Figure 4. Comparison of the apoptosis index, MVD and PCNA labeling index: 1, control; 2, $40 \mathrm{mg} / \mathrm{kg}$ of CPT-11; 3, $5 \mathrm{mg} / \mathrm{kg}$ of bevacizumab and 4, combination.

Table I. Lung metastases at day 36 after the cecal removal.

\begin{tabular}{lcccc}
\hline & \multicolumn{3}{c}{ Lung metastasis } & \\
Treatment & $(-)$ & $(+)$ & $(++)$ & P-value $\left(\chi^{2}\right.$ test $)$ \\
\hline $\begin{array}{l}\text { Control } \\
(\mathrm{n}=14)\end{array}$ & 3 & 3 & 8 & \\
$\begin{array}{l}\text { CPT-11 }(40 \mathrm{mg} / \mathrm{kg}) \\
(\mathrm{n}=16)\end{array}$ & 9 & 2 & 5 & 0.151 \\
$\begin{array}{l}\text { Bevacizumab } \\
(\mathrm{n}=11)\end{array}$ & 9 & 1 & 1 & 0.010 \\
$\begin{array}{l}\text { Combination } \\
(\mathrm{n}=11)\end{array}$ & 8 & 1 & 2 & 0.037 \\
\hline
\end{tabular}

Inhibitory effect on lung metastasis at day 35 after the cecal removal. The incidence of lung metastases in the group treated with CPT-11 appeared to be lower than that in the control group at day 35 after the cecal removal, but the difference was not significant $(\mathrm{p}=0.151)$. The incidence in the group treated with bevacizumab was significantly $(\mathrm{p}=0.010)$ lower than that in the control group. The incidence in the group treated with the combination of CPT-11 and bevacizumab was significantly $(p=0.037)$ lower than that in the control group. However, there was no difference in the incidence of lung metastasis between the bevacizumab-alone and the combination groups (Table I).

The average number of macroscopic tumor nodules on the surface of the lung was $5.5 \pm 2.4$ in the CPT-alone, $0.8 \pm 0.8$ in the bevacizumab-alone, and $2.4 \pm 1.8$ in the combination groups. A significant $(\mathrm{p}=0.024)$ difference in the number between the bevacizumab-alone group and the control group was noted. No effect in the number of tumor nodules was found from adding CPT-11 to bevacizumab (Table II).

\section{Discussion}

Based on the results of our previous preclinical study (14), we postulated that cytotoxic anticancer drugs may be coadministered with antiangiogenic agents in a clinical adjuvant setting. The antitumor effects of the antiangiogenic

Table II. The number of macroscopic lung metastases.

\begin{tabular}{|c|c|c|c|c|}
\hline $\begin{array}{l}\text { Number of } \\
\text { metastases }\end{array}$ & $\begin{array}{l}\text { Control } \\
(n=14)\end{array}$ & $\begin{array}{c}\text { CPT-11 } \\
(40 \mathrm{mg} / \mathrm{kg})\end{array}$ & $\begin{array}{l}\text { Bevacizumab } \\
\quad(\mathrm{n}=11)\end{array}$ & $\begin{array}{l}\text { Combination } \\
\quad(n=11)\end{array}$ \\
\hline 0 & 6 & 11 & 10 & 9 \\
\hline $1-10$ & 3 & 3 & 1 & 1 \\
\hline $11-20$ & 2 & 1 & 0 & 1 \\
\hline $21-30$ & 1 & 0 & 0 & 0 \\
\hline $31-40$ & 1 & 1 & 0 & 0 \\
\hline $41-50$ & 1 & 0 & 0 & 0 \\
\hline $51-$ & 0 & 0 & 0 & 0 \\
\hline Average number & $12.4 \pm 4.2$ & $5.5 \pm 2.4$ & $0.8 \pm 0.8$ & $2.4 \pm 1.8$ \\
\hline P-value: & & 0.152 & 0.024 & 0.057 \\
\hline
\end{tabular}


agents may be minimal when neovascularization has already induced metastases after removal of the primary colon cancer as often seen in the clinical postoperative situation. The present study was performed to determine the activity of antiVEGF bevacizumab combined with the topoisomerase I inhibitor, CPT-11, using colon cancer zenografts, after neovascularization was already induced around the metastases, and to provide a rationale for clinical development as adjuvant chemotherapy, containing this combination or bevacizumab combined with other cytotoxic agents.

Bevacizumab or CPT alone showed significant growth suppression of the subcutaneously implanted colon cancer in rat. Our findings showed that a combination of bevacizumab and CPT-11 was definitively more effective on tumor growth inhibition compared with each monotherapy. Biological examinations using subcutaneously implanted tumors 35 days after the beginning of treatment, demonstrated marked differences between the combination therapy of bevacizumab and CPT-11, and the control or monotherapy. The combination therapy resulted in a decrease in tumor cell proliferation (PI) and an increase in tumor cell apoptosis (AI). The MVD was significantly lower in the group containing bevacizumab than that in the group without bevacizumab. These findings suggested that the mechanism of the antitumor effect of the combination was suspected to be a synergistic induction of apoptosis through suppression in angiogenesis, in the implanted colon cancer in the subcutis. These results are consistent with a number of studies demonstrating the principle that combined therapy with bevacizumab and antineoplastic drugs resulted in a marked potentiation of antitumor activity compared with monotherapy. As a reason for this synergy, bevacizumab has been shown to augment the antitumor activity of several anticancer agents, including doxorubicin, topotecan, paclitaxel, docetaxel and gemcitabine in preclinical models $(13,16)$. It has been reported that antiangiogenic agents may induce normalizing tumor vasculature and decrease in interstitial pressure in the tumor tissue $(17,18)$, resulting in an increased transport of the anticancer drug to the tumor tissue (9).

However, our data did not demonstrate any additional effect from CPT-11 when combined with bevacizumab on the inhibition of lung metastases after neovascularization had already become induced in the resection model of the orthotopically implanted colon cancer, because the bevacizumabalone and the combination with CPT-11 showed a marked efficacy. Our study design had not expected such a definitive efficacy of bevacizumab alone. There was also no difference in the efficacy on inhibition of lung metastasis according to the time of bevacizumab administration (immediately after or from day 15 after the cecal removal) (data not shown). Our speculation for the unexpected high efficacy of bevacizumab alone is that the KM12SM was a highly-productive cell line for VEGF. Therefore, bevacizumab was thought to respond well to VEGF-induced angiogenesis and to carry an antitumor effect even after neovascularization became induced in the metastases. Bevacizumab monotherapy has been shown to be effective in highly VEGF-dependent tumors such as renal cell carcinoma (17). Moreover, it is possible that at day 15 after the tumor removal, there may have been no definitive phase of induction in neovascularization, as shown in the present study, because the time of the neovascularization in the metastases may have been affected by the condition of the tumor cell injection, for instance by the condition of cell viability. Considering that the implanted cecal tumor did not develop in some rats in the present study and that the size of the implanted cecal tumors tended to be smaller compared to those in our previous studies (data not shown), the exact time of neovascularization may be later than day 15 in our present study.

Nevertheless, the ability of the combination therapy of bevacizumab and CPT-11 to inhibit implanted tumor growth and lung metastases suggests that a particular regimen containing this combination is a promising therapeutic strategy for clinical testing in postoperative adjuvant chemotherapy. In previous clinical trials, the CPT-11 with 5-FU/LV regimens failed to demonstrate any additional efficacy to the 5-FU/LV as a postoperative adjuvant therapy against colon cancer $(18,19)$, whereas FOLFOX which is oxaliplatin added to bolus plus infusion 5-FU/LV has been shown to be superior to 5-FU/ LV (3). The present study was not designed to determine a combinant with bevacizumab for the adjuvant setting. Futher clinical studies may determine a better chemotherapeutic partner of bevacizumab after a potential curative operation for colorectal cancer.

We conclude that a beneficial effect of bevacizumab against postoperative lung metastases from implanted colon cancer may be achieved with or without CPT-11, regardless of the timing of neovacularization in the metastases. In addition, a higher efficacy in the postoperative recurrence may be expected when bevacizumab is combined with the cytotoxic agent, CPT-11, compared to bevacizumab monotherapy, against tumors with various VEGF production in clinical situations. We hope that clinical trials will be conducted to test the efficacy of bevacizumab in an adjuvant setting after the curative resection of colorectal cancer.

\section{References}

1. Greenlee RT, Murray T, Bolden S and Wingo PA: Cancer Statistics, 2000. CA Cancer J Clin 50: 7-33, 2000.

2. International multicentre pooled analysis of colon cancer trials (IMPACT) investigators: Efficacy of adjuvant fluorouracil and folinic acid in colon cancer. Lancet 345: 939-944, 1995.

3. Andre T, Boni C, Mounedji-Boudiaf L, Navarro M, Tabernero J, Hickish T, Topham C, Zaninelli M, Clingan P, Bridgewater J, Tabah-Fisch I and de Gramont A: Multicenter international study of oxaliplatin/5-fluorouracil/leucovorin in the adjuvant treatment of colon cancer (MOSAIC) investigators: Oxaliplatin, fluorouracil, and leucovorin as adjuvant treatment for colon cancer. N Engl J Med 350: 2343-2351, 2004.

4. Eskens FA: Angiogenesis inhibitors in clinical development. Where are we now and where are we going? Br J Cancer 90: 1-7, 2004.

5. Warren RS, Yuan H, Matli MR, Gillett NA and Ferrara N: Regulation by vascular endothelial growth factor of human colon cancer tumorigenesis in a mouse model of experimental liver metastasis. J Clin Invest 95: 1789-1797, 1995.

6. Takahashi Y, Ellis LM and Mai M: The angiogenic switch of human colon cancer occurs simultaneous to initiation of invasion. Oncol Rep 10: 9-13, 2003.

7. Hanrahan V, Currie MJ, Gunningham SP, Morrin HR, Scott PA, Robinson BA and Fox SB: The angiogenic switch for vascular endothelial growth factor (VEGF)-A, VEGF-B, VEGF-C and VEGF-D in the adenomacarcinoma sequence during colorectal cancer progression. J Pathol 200: 183-194, 2003.

8. Ferrara N, Hillan KJ, Gerber HP and Novotny W: Discovery and development of bevacizumab, an anti-VEGF antibody for treating cancer. Nat Rev Drug Discov 3: 391-400, 2004. 
9. Wilders H, Guetens G, De Boeck G, Verbeken E, Landuyt B, Landuyt W, de Bruijn EA and van Oosteron AT: Effect of antivascular endothelial growth factor treatment on the intratumoral uptake of CPT-11. Br J Cancer 88: 1979-1986, 2003.

10. Ogata Y, Matono K, Mizobe T, Ishibashi N, Mori S, Akagi Y, Ikeda S, Ozasa H, Murakami $\mathrm{H}$ and Shirouzu K: The expression of vascular endothelial growth factor determines the efficacy of post-operative adjuvant chemotherapy using oral fluoropyrimidines in stage 2 or 3 colorectal cancer. Oncol Rep 15: 1111-1116, 2006.

11. Des Guetz G, Uzzan P, Nicolas B, Cucherat M, Morere JF, Benamouzig R, Breau JL and Perret GY: Microvessel density and VEGF expression are prognostic factors in colorectal cancer. Meta-analysis of the literature. Br J Cancer 94: 1823-1832, 2006.

12. Kim KJ, Li B, Winer J, Armanini M, Gillett N, Phillip HS and Ferrara N: Inhibition of vascular endothelial growth factorinduced angiogenesis suppresses tumor growth in vivo. Nature 362: 841-844, 1993.

13. Gerber HP and Ferrara N: Pharmacology and pharmacodynamics of bevacizumab as monotherapy or in combination with cytotoxic therapy in preclinical studies. Cancer Res 65: 671-680, 2005.

14. Hurwitz H, Fehrenbacher L, Novotny W, Cartwright T, Hainsworth J, Heim W, Berlin J, Baron A, Griffing S, Holmgren E, Ferrara N, Fyfe G, Rogers B, Ross R and Kabbinavar F: Bevacizumab plus irinotecan, fluorouracil, and leucovorin for metastatic colorectal cancer. N Engl J Med 350: 2335-2342, 2004.

15. Ogata Y, Matono K, Nakajima M, Sasatomi T, Mizobe, T, Nagase $\mathrm{H}$ and Shirouzu K: Efficacy of the MMP inhibitor MMI270 against lung metastasis following removal of orthotopically transplanted human colon cancer in rat. Int J Cancer 118: 215-221, 2006.

16. Hedy LK, Gregory F, Deepti AS, Gershon L, Sreenivasa N, Mark K, David AT, Theodore K, Abraham D, Walter MS and Everett EV: Phase II trial of bevacizumab plus gemcitabine in patients with advanced pancreatic cancer. J Clin Oncol 23: 8033-8040, 2005.
17. Ricky TT, Yves B, Sergey VK, Frank W, Daniel JH and Rakesh KJ: Vascular normalization by vascular endothelial growth factor receptor 2 blockade induces a pressure gradient across the vasculature and improves drug penetration in tumors. Cancer Res 64: 3731-3736, 2004.

18. Jain RK: Normalizing tumor vasculature with anti-angiogenic therapy: A new paradigm for combination therapy. Nat Med 7: 987-989, 2001.

19. James CY, Leah H, Richard MS, Patrick H, Douglas JS, Suzanne LT, Seth MS, Helen XC and Steven AR: A randomized trial of bevacizumab, an anti-vascular endothelial growth factor antibody, for metastatic renal cancer. N Engl J Med 349: 427-434, 2003.

20. Saltz LB, Niedzwiecki D, Hollis D, Goldberg RM, Hantel A, Thomas JP, Fields AL and Mayer RJ: Irinotecan fluorouracil plus leucovorin is not superior to fluorouracil plus leucovorin alone as adjuvant treatment for stage III colon cancer: Results of CALGB 89803. J Clin Oncol 25: 3456-3461, 2007.

21. Ychou M, Raoul J, Douillard J, Bugat R, Mineur L, Viret F, Becouarn Y, Bouche O, Jacob J and Gourgou-Bourgade $S$ for the GI Group of the FNCLCC and the FFCD: A phase III randomized trial of LV5FU2+CPT-11 vs LV5FU2 alone in adjuvant high risk colon cancer (FNCLCC Accord 02/FFCD 9802). J Clin Oncol 23: S3502, 2005.

22. Van Cutsem E, Labianca R, Hossfeld D, Bodoky G, Roth A, Aranda E, Nordlinger B, Assadourian S, Wang K and Cunningham D (PETACC 3): Randomized phase III trial comparing infused irinotecan/5-fluorouracil (5-FU)/folinic acid (IF) versus 5-FU/FA (F) in stage III colon cancer patients. J Clin Oncol 23: S8, 2005. 